\title{
Control of brain energy supply by astrocytes
}

\section{Ross Nortley \& David Attwell}

\section{Department of Neuroscience, Physiology \& Pharmacology \\ University College London \\ Gower Street, London, WC1E 6BT, UK}

Address correspondence to David Attwell, d.attwell@ucl.ac.uk 


\begin{abstract}
Astrocytes form an anatomical bridge between the vasculature and neuronal synapses. Recent work suggests that they play a key role in regulating brain energy supply by increasing blood flow to regions where neurons are active, and setting the baseline level of blood flow. Controversy persists over whether lactate derived from astrocyte glycolysis is used to power oxidative phosphorylation in neurons, but astrocytes sustain neuronal ATP production by recycling neurotransmitter glutamate that would otherwise need to be resynthesized from glucose, and by providing a short term energy store in the form of glycogen that can be mobilised when neurons are active.
\end{abstract}

\title{
Introduction
}

The human brain is critically dependent on a constant supply of energy (almost exclusively in the form of glucose) to meet its high metabolic demands. Despite comprising only $2 \%$ of the body's mass, the brain consumes approximately $20 \%$ of its resting energy, mainly to reverse ion fluxes that underlie synaptic potentials and action potentials $[1,2,3]$, and if the energy supply of brain cells is compromised they quickly become injured or die. To ensure that the fluctuating activity-dependent energy requirements of neurons are met, the brain has evolved 'neurovascular coupling' mechanisms to regulate energy supply, which increase the blood flow to regions where neurons are active - a response termed 'functional hyperaemia' [4]. Once delivered to an active area, glucose must then be successfully transferred from the blood to brain cells, where it is used to generate ATP, converted to other forms of energy substrate (such as lactate or glutamate) or converted to the storable energy reserve glycogen $[5,6]$.

Astrocytes, with 'endfoot' processes abutting blood vessels supplying glucose, and finer processes surrounding the synapses that are the brain's major energy consumers, are potentially important regulators of brain energy supply. Surprisingly, however, there has been intense debate about the role of astrocytes, both in regulating local blood flow to power active neurons, and in transforming glucose to other molecules that are the immediate substrate for ATP production. In this review we discuss the pivotal roles that astrocytes may play in the 'flow' of energy from the circulation to brain cells. 


\section{The role of astrocyte $\left[\mathrm{Ca}^{2+}\right]_{\mathrm{i}}$ transients in adjusting brain energy supply}

Astrocytes, which are present in the brain at similar numbers to neurons [7*], are ideally positioned between the vasculature and neurons to fulfil several key roles in regulating the flow of energy to neurons, including mediation of neurovascular coupling. Astrocytes extend fine processes that ensheath neuronal synapses and more substantial 'endfeet' that wrap much of the surface of the brain vasculature. For example, astrocyte processes cover $\sim 63 \%$ of capillaries, with most of the rest of the endothelial tube being covered by pericytes (the role of which we discuss below), and $<1 \%$ of the endothelium facing clefts between these cells [8]. This topographical arrangement allows astrocytes to detect changes in neuronal activity (and hence metabolic demand) by sensing neurotransmitter release, and to relay this information to the vasculature to alter the energy supply. Classically, regulation of blood flow is achieved by vascular smooth muscle cells altering their tone to adjust the diameter of arterioles, and thus alter blood flow.

The first evidence that astrocytes can regulate arteriole diameter came from brain slices, where the Carmignoto group showed that raising astrocyte $\left[\mathrm{Ca}^{2+}\right]_{\mathrm{i}}$ with metabotropic glutamate receptor (mGluR) agonists dilated arterioles by generating a cyclooxygenase derivative of arachidonic acid [9], and the MacVicar group showed that raising astrocyte $\left[\mathrm{Ca}^{2+}\right]_{\mathrm{i}}$ by uncaging $\mathrm{Ca}^{2+}$ led to arteriole constriction (later found to become a dilation at physiological oxygen levels [10, 11]). Similarly, Nedergaard's group reported that in vivo a similar mGluR-evoked arteriole dilation occurred in response to neuronal activity and was mediated by a cyclooxygenase derivative [12]. Subsequently, debate has raged over whether the astrocyte $\left[\mathrm{Ca}^{2+}\right]_{\mathrm{i}}$ transients evoked by neuronal activity are too small, too slow, or too infrequent to have a causative role in neurovascular coupling $[13,14,15]$, and whether $\left[\mathrm{Ca}^{2+}\right]_{\mathrm{i}^{-}}$ raising mGluRs exist in adult astrocytes [16]. These arguments partly reflected the fact that conclusions were based on measuring $\mathrm{Ca}^{2+}$ signals within astrocyte cell bodies rather than in the fine processes of astrocytes near synapses which are presumably the first responders to neuronal activity, and on using bulk loading of $\mathrm{Ca}^{2+}$-sensing dyes which may go into both astrocytes and neurons thus making it hard to be certain which cell type is generating the observed $\mathrm{Ca}^{2+}$ signal $\left[17,18^{*}\right.$, reviewed in depth by 19$]$.

In addition to releasing enzyme-derived chemical messengers, neuronal activity-driven astrocyte $\left[\mathrm{Ca}^{2+}\right]_{\mathrm{i}}$ transients may also signal to blood vessels by releasing $\mathrm{K}^{+}$ions onto vessels, through 
$\mathrm{Ca}^{2+}$-activated $\mathrm{K}^{+}(\mathrm{BK})$ channels in astrocyte endfeet [20]. In the physiological voltage range increasing $\left[\mathrm{K}^{+}\right]_{\mathrm{o}}$ hyperpolarises vascular smooth muscle, by increasing the conductance of, and thus increasing outward current through, inward rectifier $\mathrm{K}^{+}$channels. This hyperpolarisation reduces $\mathrm{Ca}^{2+}$ influx through voltage-gated channels, and leads to vessel dilation. A similar mechanism has been suggested to initiate a propagating hyperpolarization along capillary endothelial cells, to send a signal instructing arterioles to dilate [21].

\section{Astrocytes mediate neurovascular coupling at the capillary level}

Despite a general focus on neurovascular coupling at the arteriole level, it was recently demonstrated that the majority of the hydraulic resistance which can be reduced to increase blood flow in the cortical vasculature is located in capillaries rather than arterioles [22]. This has shifted attention to the role of pericytes - spatially isolated contractile cells on capillaries - in controlling cerebral blood flow. Indeed, in vivo data suggest that a major fraction of the increase in blood flow evoked by neuronal activity reflects active relaxation of capillary pericytes [23, $24 *]$.

Dialysing astrocytes in brain slices with a high concentration of the rapid calcium buffer BAPTA was found to inhibit pericyte-mediated capillary dilation, while having no effect on arteriole dilation [25] (perhaps surprisingly, given previous reports $[11,12]$ of astrocyte $\left[\mathrm{Ca}^{2+}\right]$ regulating arteriole dilation), establishing a role for astrocyte $\left[\mathrm{Ca}^{2+}\right]_{\mathrm{i}}$ transients in regulating brain energy supply at the capillary level. Further pharmacological analysis demonstrated that the signalling evoked by neuronal activity to dilate the two types of vessel in the brain slices used for these experiments was different, with arterioles being dilated by nitric oxide release (presumably from interneurons), and capillaries being dilated by prostaglandin $\mathrm{E}_{2}$ which was generated from arachidonic acid derived from the sequential action (in astrocytes) of phospholipase D2, diacylglycerol lipase and cyclooxygenase 1. (We note however that other studies [11, 12] have reported that arachidonic acid metabolites such as $\mathrm{PgE}_{2}$ can also dilate arterioles, suggesting that different neurovascular coupling mechanisms may occur in different circumstances). The astrocyte $\left[\mathrm{Ca}^{2+}\right]_{\mathrm{i}}$ transients evoked by neuronal activity to initiate capillary dilation were surprisingly shown to be produced, not by mGluRs as discussed above, but by postsynaptically released ATP activating $\mathrm{P}_{2} \mathrm{X}_{1}$ receptors on astrocytes [25] (Fig. 1). Similarly, in the retina, astrocyte (Müller cell) $\left[\mathrm{Ca}^{2+}\right]_{\mathrm{i}}$ transients were found to dilate intermediate layer capillaries but 
not arterioles [26*]. It has been suggested that a low level of neuronal activity can evoke arteriole dilation in the absence of astrocyte endfoot $\left[\mathrm{Ca}^{2+}\right]_{i}$ transients, which are only detectable when a higher level of neuronal activity occurs [27] and it will be interesting to determine whether this dilation is mediated by NO, as found in [25]. If so this might imply that low levels of neuronal activity increase blood flow solely by dilating arterioles, while higher activity also dilates capillaries via astrocytes and pericytes.

The control of capillary diameter by astrocytes and pericytes may be relevant to developing strategies for preventing deleterious decreases of blood flow that occur in pathology. Both after ischaemia [23] and in epilepsy models [28] localised constrictions of capillaries produced by pericytes reduce local blood flow, which will contribute to neurodegeneration. An important issue for the future will be whether the normal control of pericyte tone by astrocytes can be harnessed therapeutically to maintain a normal energy supply to the tissue in such situations.

\section{Tonic regulation of cerebral blood flow by astrocytes}

Changes of blood flow evoked by neuronal activity, in part generated through astrocytes as discussed above, initiate the signals detected by the widely used BOLD fMRI (blood oxygen level dependent functional magnetic resonance imaging) technique. However, a tonic regulation of cerebral blood flow by astrocytes may be of equal importance for brain function. Introducing the calcium chelator BAPTA into astrocytes in brain slices was found to produce a constriction of arterioles that was prevented by blocking cyclooxygenase 1 , but was unaffected by blocking neuronal activity with TTX [29]. Thus, either the mean resting level of astrocyte $\left[\mathrm{Ca}^{2+}\right]_{\mathrm{i}}$ or $\left[\mathrm{Ca}^{2+}\right]_{\mathrm{i}}$ transients generated independently of action potentials may tonically activate the prostaglandin-mediated pathway described above [25], although it is surprising that in this case it is affecting arterioles rather than capillary pericytes. Recently, astrocyte $\left[\mathrm{Ca}^{2+}\right]_{\mathrm{i}}$ transients generated independently of action potential evoked transmitter release were characterised as resulting from bursts of $\mathrm{Ca}^{2+}$ efflux through the mitochondrial permeability transition pore [30**]. Although these transients occur in TTX, their rate was raised when neuronal activity was increased by blocking synaptic inhibition with picrotoxin. This may occur because the mitochondrial permeability transition pore opens more when demand for oxidative phosphorylation is greater during neuronal activity [30**]. If so, this would provide another astrocyte-mediated 
mechanism to increase the supply of glucose and oxygen substrates to oxidative phosphorylation when needed.

Another possible way in which astrocytes may play a key role in regulating cerebral blood flow tonically is in response to changes of $\mathrm{CO}_{2}$ level $\left[31^{*}\right]$. When $\left[\mathrm{CO}_{2}\right]$ rises, astrocyte $\left[\mathrm{Ca}^{2+}\right]_{\mathrm{i}}$ rises (possibly as a result of activation of $\mathrm{Na}^{+} / \mathrm{HCO}_{3}^{--}$co-transport into the cell followed by a slowing or reversal of $\mathrm{Na} / \mathrm{Ca}^{2+}$ exchange [32]), and this activates cyclooxygenase 1 (COX1) to generate vasodilating prostaglandin $\mathrm{E}_{2}$. The enzyme downstream of $\mathrm{COX} 1$ that synthesizes prostaglandin $\mathrm{E}_{2}$ depends for its activity on glutathione, which can fall in concentration in disease or old age, suggesting a possible mechanism by which cerebral blood flow and energy supply might be reduced in these conditions [31*].

\section{Signalling from the vasculature to neurons via astrocytes}

Remarkably, communication between neurons and the vasculature via astrocytes may be bidirectional. Increasing the flow and pressure within arterioles in brain slices has been found to constrict the arterioles and raise $\left[\mathrm{Ca}^{2+}\right]_{\mathrm{i}}$ in astrocyte processes via activation of TRPV4 channels [33]. This astrocyte $\left[\mathrm{Ca}^{2+}\right]_{i}$ rise leads, perhaps via release of the gliotransmitter ATP which is converted extracellularly into adenosine, to increased spiking activity of inhibitory interneurons and a hyperpolarization and decreased spiking activity of pyramidal neurons [33]. At present the functional significance of this pathway for matching neuronal activity to supply of energy substrates is uncertain.

\section{Astrocyte control of neuronal energy use?}

After arriving in the blood, glucose crosses cerebral vascular endothelial cells via facilitative (sodium-independent) GLUT1 glucose transporters, before uptake mainly via GLUT1 and GLUT3 into glial cells and neurons, respectively (Fig. 1). Here, it undergoes conversion to glucose-6-phosphate by hexokinase, before being processed by the glycolysis pathway to generate ATP and pyruvate, by the pentose phosphate pathway to generate NADPH, or (in astrocytes only) being converted to the energy store glycogen [34]. Pyruvate generated by glycolysis can either be used directly (in the same cell) by oxidative phosphorylation to generate a much larger amount of ATP per glucose than glycolysis provides, or can be converted to lactate and exported from the cell, either to be lost from the brain or to be taken up into another cell and converted back to pyruvate to be used in oxidative phosphorylation [34]. 
Neurons are the principal consumers of brain energy ( $\sim 80 \%)$, which they use mainly to reverse the ion entry generating synaptic and action potentials $[2,3]$. The vasculature-wrapping morphology of astrocytes described above might suggest that glucose may be initially transferred from blood vessels into astrocytes before being distributed to neurons through the astrocyte cytoplasm (although modelling based on transporter data [35] has indicated that most glucose could in fact reach neurons by diffusion through the extracellular space: Fig. 1), and even through astrocyte-astrocyte gap junctions to more distant regions [36]. The influential astrocyte-neuron lactate shuttle (ANLS) hypothesis [37] proposed an appealing mechanism by which neuronal activity could thus be linked to energy supply. When neurons are active and releasing glutamate, uptake of the glutamate into astrocytes and its subsequent conversion to glutamine were postulated to trigger glycolysis in the astrocytes, generating lactate (made from pyruvate via astrocytic lactate dehydrogenase (LDH): Fig. 1) that could then be exported to neurons and used as fuel for oxidative phosphorylation (after conversion by neuronal LDH back to pyruvate). Export of the lactate would occur via monocarboxylate transporters (MCTs) or perhaps a channel-based mechanism [38].

The ANLS hypothesis was based partly on the affinities of the LDH and MCT isoforms present in neurons and astrocytes being suited to transfer lactate from astrocytes to neurons, however the overall direction of the lactate flux is determined by concentrations of the reactants involved in the reactions catalysed by LDH and not by the MCT and LDH affinities [39]. The ANLS hypothesis was also based on the notion that astrocytes are much more glycolytic than neurons, which were hypothesised to generate ATP by oxidative phosphorylation using astrocyte-derived lactate as a substrate. Indeed, 6phosphofructo-2-kinase/fructose-2,6-biphosphatase 3 (Pfkfb3), which promotes glycolysis by converting fructose-6-phosphate to fructose-2,6-bisphosphate which allosterically activates the ratelimiting glycolytic enzyme phosphofructokinase, is more highly expressed in astrocytes than in neurons [40, 41*]. Further downstream in glycolysis, astrocytes and neurons differ in the splice variant they express of the enzyme pyruvate kinase, which generates pyruvate from phosphoenolpyruvate, as a result of which it has been suggested that astrocytes are more able than neurons to regulate their glycolytic rate to match the prevailing energetic conditions [42]. Furthermore, in cultured cells, the activity of pyruvate dehydrogenase (PDH), which regulates entry of pyruvate into the TCA cycle, is more inhibited 
by phosphorylation in astrocytes than in neurons, thus diverting pyruvate from the TCA cycle and promoting lactate formation $[42,43]$.

Despite these data suggesting that astrocytes use glycolysis more than neurons, it certainly is not the case that all astrocyte ATP generation is via glycolysis, while all neuronal ATP is produced by lactate-fuelled oxidative phosphorylation in mitochondria. Tracing a fluorescent glucose analogue has suggested that neuronal activity promotes its uptake mainly into neurons rather than astrocytes, consistent with neurons being important users of glycolysis [44]. Astrocytes have mitochondria even in their fine processes [45], and indeed neuronal activity localises mitochondria at positions in astrocyte processes that are near synapses $[46,47]$, presumably in order to power glutamate uptake that occurs there. Furthermore, if neuronal ATP were made using oxidative phosphorylation fuelled by astrocytederived lactate under all circumstances, then blocking LDH would inhibit the rise in oxygen consumption evoked by stimulating neuronal activity, but in brain slices blocking LDH has no such effect [48]. One might ask why the effect of an astrocyte-selective inducible deletion or knock-down of MCTs or LDH has not yet been reported (as has been performed for oligodendrocyte-axon energetic interactions [49]), as an incisive test of the idea that lactate trafficking from astrocytes to neurons is crucial for neuronal function. In fact when this was done for MCT1 in astrocytes or MCT2 in neurons [50], although it inhibited long-term memory, it had no effect on memory acquisition or short-term memory, arguing against an obligatory role for lactate transport via MCT1 and MCT2 to power neuronal function on a rapid time scale (but see below).

\section{Astrocytes and long-term energy reserves}

Conversion of glucose to glycogen in astrocytes provides a limited reserve of energy, which may be important for sustaining neuronal function in periods of intense energy use, which may perhaps

occur during long-term term memory formation [50], since this is inhibited by preventing glycogenolysis or MCT-mediated transfer of lactate to neurons. However, estimates from experiments blocking ATP synthesis suggest that glycogen can sustain neuronal function for only a few minutes (even in brain slices where energy use is reduced compared to the in vivo situation) [51]. A more significant long-term contribution of astrocytes to brain energetics lies in the fact that they take up over $90 \%$ of the glutamate released as a neurotransmitter - a molar amount comparable to the amount of 
glucose used by the brain [52]. If this glutamate were lost to the blood, then it would be necessary to approximately double the glucose supply to the brain in order to provide carbon skeletons for glutamate synthesis, in addition to the glucose needed as a substrate for ATP production.

\section{Conclusions}

Although the jury is still out on the direction of lactate trafficking between neurons and astrocytes [34], an important energetic role for astrocytes is becoming clear at the level of blood flow regulation. Studying how pathology disrupts the astrocyte-based mechanisms regulating brain energy supply will be an important focus of future work.

\section{Acknowledgements}

This work was supported by a Senior Investigator Award to DA from the Wellcome Trust (099222/Z/12/Z), an Advanced Investigator Award to DA from the European Research Council (BrainPower), and a Leonard Wolfson 4 year PhD studentship to RN. The funders had no role in the content of the review. 


\section{References}

1. Mink JW, Blumenschine RJ, Adams DB: Ratio of central nervous system to body metabolism in vertebrates: its constancy and functional basis. Am J Physiol 1981, 240:203-212.

2. Attwell D, Laughlin SB: An energy budget for signalling in the grey matter of the brain. J Cereb Blood Flow Metab 2001, 21:1133-1145.

3. Harris JJ, Jolivet R, Attwell D: Synaptic energy use and supply. 2012 Neuron 75:762-777.

4. Attwell D, Buchan A, Charpak S, Lauritzen M, MacVicar BA, Newman EA: Glial and neuronal control of blood flow. 2010 Nature 468:232-243.

5. Waitt AE, Reed L, Ransom BR, Brown AM: Emerging roles for glycogen in the CNS. 2017 Front Mol Neurosci 10:73 doi: 10.3389/fnmol.2017.00073.

6. McKenna MC, Stridh MH, McNair LF, Sonnewald U, Waagepetersen HS, Schousboe A: Glutamate oxidation in astrocytes: Roles of glutamate dehydrogenase and aminotransferases. $2016 \mathrm{~J}$ Neurosci Res 94:1561-1571.

7. *von Bartheld CS, Bahney J, Herculano-Houzel S: The search for true numbers of neurons and glial cells in the human brain: A review of 150 years of cell counting. $2016 \mathrm{~J}$ Comp Neurol 524:3865-3895.

A fascinating account of how estimates of the relative numbers of neurons and glial cells have changed over the years, and how most textbook statements on the issue are wrong.

8. Mathiisen TM, Lehre KP, Danbolt NC, Ottersen OP: The perivascular astroglial sheath provides a complete covering of the brain microvessels: an electron microscopic 3D reconstruction. 2010 Glia 58:1094-1103.

9. Zonta M, Angulo MC, Gobbo S, Rosengarten B, Hossmann KA, Pozzan T, Carmignoto G. Neuronto-astrocyte signalling is central to the dynamic control of brain microcirculation. $2003 \mathrm{Nat}$ Neurosci 6:43-50.

10. Mulligan SJ, MacVicar BA. Calcium transients in astrocyte endfeet cause cerebrovascular constrictions. 2004 Nature 431:195-199.

11. Gordon GR, Choi HB, Rungta RL, Ellis-Davies GC, MacVicar BA. Brain metabolism dictates the polarity of astrocyte control over arterioles. 2008 Nature 456:745-749. 
12. Takano, T., Tian GF, Peng W, Lou N, Libionka W, Han X, Nedergaard M. Astrocyte mediated control of cerebral blood flow. 2006 Nat Neurosci 9:260-267.

13. Winship IR, Plaa N, Murphy TH. Rapid astrocyte calcium signals correlate with neuronal activity and onset of the hemodynamic response in vivo. $2007 \mathrm{~J}$ Neurosci 27:6268-6272.

14. Nizar K, Uhlirova H, Tian P, Saisan PA, Cheng Q, Reznichenko L, Weldy KL, Steed TC, Sridhar VB, MacDonald CL, et al. In vivo stimulus-induced vasodilation occurs without $\mathrm{IP}_{3}$ receptor activation and may precede astrocytic calcium increase. 2013 J Neurosci 33:8411-8422.

15. Lind BL, Brazhe AR, Jessen SB, Tan FC, Lauritzen MJ Rapid stimulus-evoked astrocyte Ca ${ }^{2+}$ elevations and hemodynamic responses in mouse somatosensory cortex in vivo. 2013 Proc Natl Acad Sci USA 110:E4678-E4687.

16. Sun W, McConnell E, Pare JF, Xu Q, Chen M, Peng W, Lovatt D, Han X, Smith Y, Nedergaard M. Glutamate-dependent neuroglial calcium signalling differs between young and adult brain. 2013 Science 339:197-200.

17. Reeves AM, Shigetomi E, Khakh BS. Bulk loading of calcium indicator dyes to study astrocyte physiology: key limitations and improvements using morphological maps. $2011 \mathrm{~J}$ Neurosci 31:9353-9358.

18. *Otsu Y, Couchman K, Lyons DG, Collot M, Agarwal A, Mallet JM, Pfrieger FW, Bergles DE, Charpak S. Calcium dynamics in astrocyte processes during neurovascular coupling. 2015 Nat Neurosci 18:210-218.

Re-established the possibility of astrocytes mediating neurovascular coupling, by using a geneticallyencoded calcium indicator to show that astrocyte process $\left[\mathrm{Ca}^{2+}\right]_{i}$ rises when neurons are active with kinetics fast enough to be a signal to increase blood flow.

19. Bazargani N, Attwell D. Astrocyte calcium signalling: the third wave. 2016 Nat Neurosci 19:182189.

20. Filosa JA, Bonev AD, Straub SV, Meredith AL, Wilkerson MK, Aldrich RW, Nelson MT. Local potassium signalling couples neuronal activity to vasodilation in the brain. 2006 Nat Neurosci. 9:1397-1403. 
21. Longden TA, Dabertrand F, Koide M, Gonzales AL, Tykocki NR, Brayden JE, Hill-Eubanks D, Nelson MT. Capillary $\mathbf{K}^{+}$-sensing initiates retrograde hyperpolarization to increase local cerebral blood flow. 2017 Nat Neurosci doi: 10.1038/nn.4533. [Epub ahead of print].

22. Blinder P, Tsai PS, Kaufhold JP, Knutsen PM, Suhl H, Kleinfeld D. The cortical angiome: an interconnected vascular network with noncolumnar patterns of blood flow. 2013 Nat Neurosci 16:889-897.

23. Hall CN, Reynell C, Gesslein B, Hamilton NB, Mishra A, Sutherland BA, O'Farrell FM, Buchan AM, Lauritzen M, Attwell D. Capillary pericytes regulate cerebral blood flow in health and disease. 2014 Nature 508:55-60.

24. *Kisler K, Nelson AR, Rege SV, Ramanathan A, Wang Y, Ahuja A, Lazic D, Tsai PS, Zhao Z, Zhou Y, et al. Pericyte degeneration leads to neurovascular uncoupling and limits oxygen supply to brain. 2017 Nat Neurosci 20:406-416.

Highlights the potential role of damage to capillary pericytes in pathology.

25. Mishra A, Reynolds JP, Chen Y, Gourine AV, Rusakov DA, Attwell D. Astrocytes mediate neurovascular signalling to capillary pericytes but not to arterioles. 2016 Nat Neurosci 12:16191627.

26. *Biesecker KR, Srienc AI, Shimoda AM, Agarwal A, Bergles DE, Kofuji P, Newman EA Glial cell calcium signalling mediated capillary regulation of blood flow in the retina. $2016 \mathrm{~J}$ Neurosci 36:9435-9445.

Uses a genetically-encoded calcium indicator to demonstrate in the retina that astrocyte (Müller cell) $\left[\mathrm{Ca}^{2+}\right]_{\mathrm{i}}$ transients dilate capillaries but not arterioles.

27. Institoris Á, Rosenegger DG, Gordon GR. Arteriole dilation to synaptic activation that is subthreshold to astrocyte endfoot $\mathbf{C a}^{2+}$ transients. 2015 J Cereb Blood Flow Metab 35:1411-1415.

28. Leal-Campanario R, Alarcon-Martinez L, Rieiro H, Martinez-Conde S, Alarcon-Martinez T, Zhao X, LaMee J, Popp PJ, Calhoun ME, Arribas JI, et al. Abnormal capillary vasodynamics contribute to ictal neurodegeneration in epilepsy. 2017 Science Rep. 7:43276 doi: 10.1038/srep43276. 
29. Rosenegger DG, Tran CH, Wamsteeker Cusulin JI, Gordon GR. Tonic local brain blood flow control by astrocytes independent of phasic neurovascular coupling. 2015 J Neurosci 35:1346313474.

30. **Agarwal A, Wu PH, Hughes EG, Fukaya M, Tischfield MA, Langseth AJ, Wirtz D, Bergles DE. Transient opening of the mitochondrial permeability transition pore induces microdomain calcium transients in astrocyte processes. 2017 Neuron 93:587-605.

Demonstrates a novel mechanism by which astrocyte $\left[\mathrm{Ca}^{2+}\right]_{\mathrm{i}}$ transients are generated.

31. *Howarth C, Sutherland B, Choi HB, Martin C, Lind BL, Khennouf L, LeDue JM, Pakan JM, Ko RW, Ellis-Davies G, et al. A critical role for astrocytes in hypercapnic vasodilation in brain. 2017 J Neurosci 37:2403-2414.

Reveals a mechanism by which $\mathrm{CO}_{2}$ increases cerebral blood flow, with implications for how blood flow may decrease in old age or pathology.

32. Turovsky E, Theparambil SM, Kasymov V, Deitmer JW, Del Arroyo AG, Ackland GL, Corneveaux JJ, Allen AN, Huentelman MJ, Kasparov S, et al. Mechanisms of $\mathrm{CO}_{2} / \mathbf{H}^{+}$sensitivity of astrocytes. $J$ Neurosci 36:10750-10758.

33. Kim KJ, Ramiro Diaz J, Iddings JA, Filosa JA. Vasculo-neuronal coupling: retrograde vascular communication to brain neurons. 2015 J Neurosci 36:12624-12639.

34. Mergenthaler P, Lindauer U, Dienel GA, Meisel A. Sugar for the brain: the role of glucoose in physiological and pathological brain function. 2013 Trends Neurosci 36:587-597.

35. Simpson IA, Carruthers A, Vannucci SJ. Supply and demand in cerebral energy metabolism: the role of nutrient transporters. 2007 J Cereb Blood Flow Metab 27:1766-1791.

36. Rouach N, Koulakoff A, Abudara V, Willecke K, Giaume C. Astroglial metabolic networks sustain hippocampal synaptic transmission. 2008 Science 322:1551-1555.

37. Pellerin L, Magistretti PJ. Sweet sixteen for ANLS. 2012 J Cereb Blood Flow Metab 32:1152-1166.

38. Sotelo-Hitschfeld T, Niemeyer MI, Mächler P, Ruminot I, Lerchundi R, Wyss MT, Stobart J, Fernández-Moncada I, Valdebenito R, Garrido-Gerter P, et al. Channel-mediated lactate release by $\mathrm{K}^{+}$-stimulated astrocytes. $2015 \mathrm{~J}$ Neurosci 35:4168-4178. 
39. Bak LK, Schousboe A. Misconceptions regarding basic thermodynamics and enzyme kinetics have led to erroneous conclusions regarding the metabolic importance of lactate dehydrogenase isoenzyme expression. 2017 J Neurosci Res doi: 10.1002/jnr.23994. [Epub ahead of print]

40. Herrero-Mendez A, Almeida A, Fernández E, Maestre C, Moncada S, Bolaños JP. The bioenergetic and antioxidant status of neurons is controlled by continuous degradation of a key glycolytic enzyme by APC/C-Cdh1. 2009 Nat Cell Biol 11:747-752.

41. *Zhang Y, Sloan SA, Clarke LE, Caneda C, Plaza CA, Blumenthal PD, Vogel H, Steinberg GK5, Edwards MS, Li G, et al. Purification and characterization of progenitor and mature human astrocytes reveals transcriptional and functional differences with mouse. 2016 Neuron 89:37-53.

A unique resource for assessing protein expression in astrocytes.

42. Zhang Y, Chen K, Sloan SA, Bennett ML, Scholze AR, O'Keeffe S, Phatnani HP, Guarnieri P, Caneda C, Ruderisch N, et al. An RNA-sequencing transcriptome and splicing database of glia, neurons, and vascular cells of the cerebral cortex. $2014 \mathrm{~J}$ Neurosci 34:11929-11947.

43. Halim ND, Mcfate T, Mohyeldin A, Okagaki P, Korotchkina LG, Patel MS, Jeoung NH, Harris RA, Schell MJ, Verma A. Phosphorylation status of pyruvate dehydrogenase distinguishes metabolic phenotypes of cultured rat brain astrocytes and neurons. 2010 Glia 58:1168-1176.

44. Lundgaard I, Li B, Xie L, Kang H, Sanggaard S, Haswell JD, Sun W, Goldman S, Blekot S, Nielsen $M$, et al. Direct neuronal glucose uptake heralds activity-dependent increases in cerebral metabolism. 2015 Nat Comm 6:6807 doi: 10.1038/ncomms7807.

45. Derouiche A, Haseleu J, Korf HW. Fine astrocyte processes contain very small mitochondria: glial ooxidative metabolism may fuel transmitter metabolism. 2015 Neurochem Res 40:2402-2413.

46. Jackson JG, O'Donnell JC, Takano H, Coulter DA, Robinson MB. Neuronal activity and glutamate uptake decrease mitochondrial mobility in astrocytes and position mitochondria near glutamate transporters. 2014 J Neurosci 34:1613-1624.

47. Stephen TL, Higgs NF, Sheehan DF, Al Awabdh S, López-Doménech G, Arancibia-Carcamo IL, Kittler JT. Miro1 regulates activity-driven positioning of mitochondria within astrocytic 
processes apposed to synapses to regulate intracellular calcium signaling. $2015 \mathrm{~J}$ Neurosci 35:15996-16011.

48. Hall CN, Klein-Flügge MC, Howarth C, Attwell D. Oxidative phosphorylation, not glycolysis, powers presynaptic and postsynaptic mechanisms underlying brain information processing. 2012 J Neurosci 32:8940-8951.

49. Lee Y, Morrison BM, Li Y, Lengacher S, Farah MH, Hoffman PN, Liu Y, Tsingalia A, Jin L, Zhang PW, et al. Oligodendroglia metabolically support axons and contribute to neurodegeneration. 2012 Nature 487:443-448.

50. Suzuki A, Stern SA, Bozdagi O, Huntley GW, Walker RH, Magistretti PJ, Alberini CM. Astrocyteneuron lactate transport is required for long-term memory formation. 2011 Cell 144:810-823.

51. Allen NJ, Káradóttir R, Attwell D. A preferential role for glycolysis in preventing the anoxic depolarization of rat hippocampal area CA1 pyramidal cells. 2005 J Neurosci 25:848-859.

52. Marcaggi P, Attwell D. Role of glial amino acid transporters in synaptic transmission and brain energetics. 2004 Glia 47:217-225. 


\section{Figure 1. The role of astrocytes in regulating brain energy supply.}

Astrocytes regulate both glucose supply in the blood and the use of that glucose to make ATP in different cell types. On the right a capillary provides glucose (and oxygen) in the blood. This nutrient supply can be regulated by changes in the tone of contractile pericytes (or smooth muscle around upstream arterioles). Pericytes dilate capillaries when astrocytes experience a rise in $\left[\mathrm{Ca}^{2+}\right]_{\mathrm{i}}$, for example when ATP released from postsynaptic neurons (stimulated by presynaptic glutamate release acting on AMPARs) activates astrocyte $\mathrm{P} 2 \mathrm{X}_{1}$ receptors [25]. This activates an enzyme cascade that leads to the release of prostaglandin $E_{2}$, which dilates pericytes. Glucose leaves the capillary by transport on GLUT1, and then diffuses extracellularly to neurons and astrocytes where it is taken up by GLUT3 or GLUT1, respectively, and then converted by hexokinase to G6P, a precursor for glycolysis and for glycogen formation. Glycolysis generates ATP, and either pyruvate to enter the tricarboxylic acid cycle and oxidative phosphorylation in mitochondria or lactate (via LDH), which can be exported from the cell by monocarboxylate transporters (MCT). It is often assumed that lactate passes from astrocytes to neurons, but this is controversial [34]. Once lactate is taken up into neurons by an MCT, it is converted back to pyruvate by LDH and is processed by the citric acid cycle and oxidative phosphorylation in mitochondria. The ATP thus generated is used mainly for powering the sodium/potassium pump.

Abbreviations: Pre: presynaptic terminal; Post: postsynaptic terminal; Glu: glutamate; AMPAR: AMPA receptor; $\mathrm{P}_{2}$ : a $\mathrm{Ca}^{2+}$-permeable receptor for ATP; PLD2: phospholipase D2; PA: phosphatidic acid; DAG: diacylglycerol; $\mathrm{COX} 1$ : cyclooxygenase 1; $\mathrm{PGH}_{2}$ : prostaglandin $\mathrm{H}_{2} ; \mathrm{PGE}_{2}$ : prostaglandin $\mathrm{E}_{2} ; \mathrm{EP}_{4}$ : receptor for $\mathrm{PGE}_{2}$; GLUT1: glucose transporter 1; GLUT3: glucose transporter 3; HK: hexokinase; G6P: glucose-6-phosphate; MCT: monocarboxylate transporter; LDH: lactate dehydrogenase; mito: mitochondrion. 


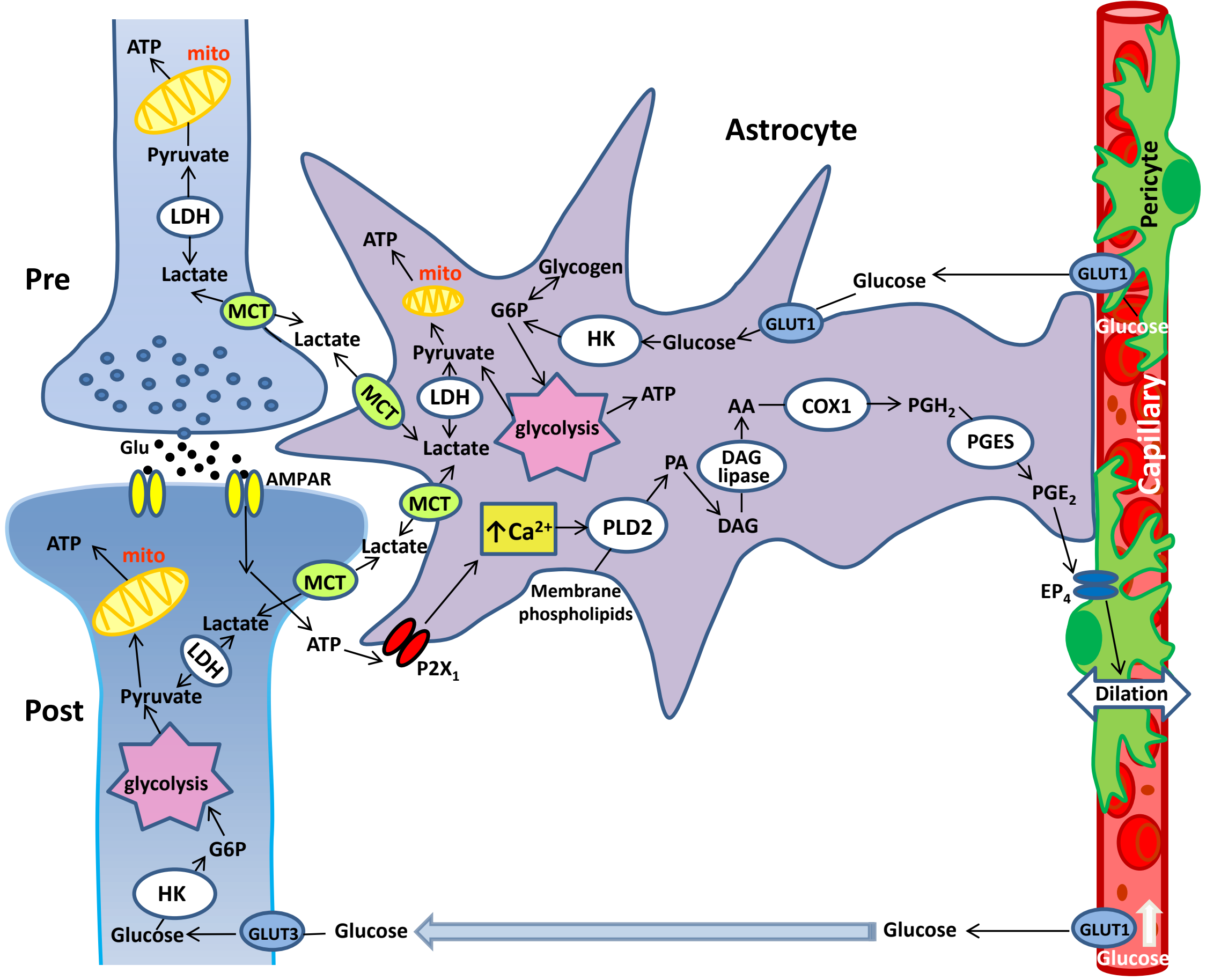

